\title{
Knockdown of MMP-1 inhibits the progression of colorectal cancer by suppressing the PI3K/Akt/c-myc signaling pathway and EMT
}

\author{
KAI WANG, JIANBAO ZHENG, JUNHUI YU, YUNHUA WU, JING GUO, ZHENGSHUI XU and XUEJUN SUN
}

Department of General Surgery, The First Affiliated Hospital of Xi'an Jiaotong University, Xi'an, Shaanxi 710061, P.R. China

Received August 7, 2019; Accepted January 14, 2020

DOI: $10.3892 /$ or.2020.7490

\begin{abstract}
The present study aimed to investigate the role of matrix metalloproteinase-1 (MMP-1) in the development of colorectal cancer and reveal the mechanism underlying this progression. Bioinformatics methods and a public dataset were first used to analyze MMP-1 gene expression in a public dataset. MMP-1 expression in colorectal cancer patients was assessed by immunohistochemistry; its association with clinicopathological parameters and its significance for prognosis were analyzed. Then proliferation, scratch and Transwell assays and a xenograft model were used to assess the change in malignant behavior in cells transfected with an MMP-1 shRNA. Changes involved in epithelial-mesenchymal transition (EMT) and the Akt signaling pathway were detected by western blotting. According to the results, MMP-1 expression was higher in colorectal cancer tissues than it was in matched adjacent noncancerous tissues, and its high expression was significantly related to lymphatic metastasis as well as TNM stage $(\mathrm{P}<0.05)$. Downregulation of MMP-1 expression inhibited the progression of colorectal cancer in vitro and in vivo. Furthermore, after the cells were stably transfected with MMP-1 shRNA, the expression of N-cadherin, vimentin and Twist1 decreased while that of E-cadherin increased. The expression of p-Akt and c-Myc also decreased. In conclusion, MMP-1 may promote malignant behavior in colorectal cancer via EMT and the Akt signaling pathway.
\end{abstract}

\section{Introduction}

Colorectal cancer is a worldwide problem, and among solid cancers, it has a significantly high incidence and mortality rate (1). In the wake of learning about the increasing importance of risk factors such as an unbalanced diet, the incidence and mortality rate have been decreasing for decades (2).

Correspondence to: Professor Xuejun Sun, Department of General Surgery, The First Affiliated Hospital of Xi'an Jiaotong University, 277 West Yanta Road, Xi'an, Shaanxi 710061, P.R. China

E-mail: sunxy@mail.xjtu.edu.cn

Key words: colorectal cancer, MMP-1, PI3K/Akt, EMT
Nevertheless, as the fifth most diagnosed cancer, colorectal cancer is one of the leading causes of cancer-related deaths in China (3). The 5-year survival rate for colorectal cancer patients can increase to $90 \%$ if diagnosed at an early stage. However, this rate will decrease to $70.4 \%$ with lymph node metastasis or invasion to adjacent organs, and it will further decrease to $12.5 \%$ when the cancer cells invade distant organs (4). As a result, the search for new genes that promote the progression of colorectal cancer may contribute to anticancer therapies.

The matrix metalloproteinases (MMPs) are a family of zinc-containing endopeptidases composed of 25 members (including collagenases, gelatinases and stromelysins) that contribute greatly to the physiological and pathological extracellular matrix remodelling (5). Their roles in the progression of cancer have been explained by the degradation of the extracellular matrix $(6,7)$. However, increasing evidence has revealed that the function of MMPs is not confined to breaking down the ECM; they can also degrade many other biomacromolecules (8). Matrix metalloproteinase-1 (MMP-1), also known as collagenase-1, has been revealed to play a significant role in the pathological progression of many cancers. The upregulation of MMP-1 has been revealed to be involved in the incidence or invasion of diverse cancers, including bladder (9), prostate (10), gastric (11), pancreatic (12) and melanoma (13). A recent study indicated that the significance of MMP-1 could make it a potential therapeutic target in lung adenocarcinoma (14). Some previous studies have suggested that MMP-1 may be expressed at a high level in colorectal cancer $(15,16)$. However, the precise mechanism by which MMP-1 regulates the progression of colorectal cancer is still unexplored.

Epithelial-mesenchymal transformation (EMT) is a common transversion by which the cells with an epithelial-like phenotype change to slender cells with a mesenchymal-like phenotype (17). EMT occurs in the progression of many pathologies, and it is followed by the attenuated capability of adhesion and absence of apico-basal polarity (18). The abated adherence function contributes to the invasion and metastasis of solid tumors (19), and it is accompanied by the weakened expression of E-cadherin and reinforced expression of vimentin (17). The latest investigations demonstrated that MMPs (MMP-2, MMP-7 and MMP-9) play a significant role in EMT in tumors (20-24). However, the relationship between MMP-1 and EMT is still unexplored. 
In the present study, the relationship between the expression levels of MMP-1 and the prognosis of colorectal cancer was investigated. The underlying molecular mechanism by which MMP-1 promotes the advancement of colorectal cancer was further explored.

\section{Materials and methods}

Data source. A first dataset with access number NM_002421 was obtained from the UCSC database (https://genome.ucsc. edu/). The dataset contained 24 samples of normal colorectal tissues and 81 samples of colorectal cancer tissues. The next dataset with the access number X05231 contained 18 samples of colorectal cancer and 18 samples of matched adjacent non-tumor tissues. Then, the last dataset, which included 22 normal colorectal tissues and 215 colorectal sample tissues, was obtained using the Cancer Genome Atlas (TCGA) database (http://tcga-data.nci.nih.gov/tcga/). The expression levels of MMP-1 in these datasets were statistically analyzed.

Cell culture. The following human colorectal cancer cell lines were obtained from Sangon Biotech Co., Ltd.: Lovo, HT-29, SW-480, HCT-116, Caco-2 and SW-620. All of these cells were cultured at $37^{\circ} \mathrm{C}$ and $5 \% \mathrm{CO}_{2}$ in Dulbecco's modified Eagle's medium (DMEM; Gibco; Thermo Fisher Scientific, Inc.) containing 100 units $/ \mathrm{ml}$ penicillin, 100 units $/ \mathrm{ml}$ streptomycin and $10 \%$ foetal bovine serum (FBS; HyClone; GE Healthcare Life Sciences); the FBS was inactivated by incubating at a constant $56^{\circ} \mathrm{C}$ for $30 \mathrm{~min}$.

Patients and specimens. We collected 49 paraffin-embedded colorectal cancer tissues and matched adjacent noncancerous tissues from patients with colorectal cancer from the First Affiliated Hospital of Xi'an Jiaotong University from January 2014 to December 2015. All patients were diagnosed with primary colorectal cancer without distant metastases. All patients underwent surgery for the first time for the treatment of colorectal cancer. No patients received radiotherapy or chemotherapy before the operation. All patients provided consent for the use of their samples, and this use was approved by the Institute Research Medical Ethics Committee of the First Affiliated Hospital of Xi'an Jiaotong University.

Transfection with shRNA. An RNA interference lentivirus containing a puromycin marker from GeneChem, Inc. was obtained; the vector can steadily downregulate the expression of MMP-1. The targeted sequence of the shRNA was UGA ACAUCACCACUGAAGGUGUAGC (25), and its efficiency was demonstrated by western blotting. A control lentivirus that did not target anything, but carried a puromycin marker as well, was also obtained. In the present study, HT-29 and SW-480 cells were infected with shRNA lentiviruses targeting MMP-1. Successfully transfected cells were selected in the aforementioned DMEM containing $1 \mu \mathrm{g} / \mathrm{ml}$ puromycin and were then expanded for the subsequent experiments.

Cell proliferative assay. Cell proliferation was assessed via a Cell Counting Kit-8 (CCK-8; Beyotime Institute of Biotechnology). A total of 2,000 cells in $200 \mu 1$ of medium were placed in each well of 96-well plates and were cultured in the abovementioned complete medium. At the instructed time-point, $20 \mu \mathrm{l}$ of CCK- 8 was added to each well, which was followed by $2 \mathrm{~h}$ of incubation. Then, the OD 450 absorbance was detected by a microplate reader (BioTek Instruments, Inc.).

Colony formation assay. Cell colony formation was examined by a colony formation assay. A total of 2,000 cells in complete medium were inoculated in $60-\mathrm{mm}$ plates and were then incubated at $37^{\circ} \mathrm{C}$ in a $5 \% \mathrm{CO}_{2}$ environment for two weeks. Cells were dyed with $0.2 \%$ dissolved crystal violet after fixation with methanol for half an hour. The images were obtained using a chemiluminescence imager (Bio-Rad Raboratories, Inc.). Then, the number of colonies that contained $>50$ cells were counted. Each assay was repeated three times.

RNA-isolation and real-time PCR. TRIzol reagent (SigmaAldrich; Merck KGaA) was used to purify the total RNA from cells according to the manufacturer's instructions. Then, the total RNA was reverse-transcribed using SYBR Green (Takara Biotechnology Co., Ltd.) and an ABI 7500 instrument (Thermo Fisher Scientific, Inc.). Primers were designed according to the reported sequences (26): MMP-1 forward, 5'-AAATGCAGG AATTCTTTGGG-3' and reverse, 5'-ATGGTCCACATCTGC TCTTG-3'; $\beta$-actin forward, 5'-TGGCACCCAGCACAATG AA-3' and reverse, 5'-CTAAG TCATAGTCCGCCTAGAAG CA-3'. The real-time PCR data were normalized to endogenous levels and were carried out in triplicate.

Immunohistochemistry (IHC). Tissue samples were formalin-fixed and paraffin-embedded, sliced into 4- $\mu \mathrm{m}$ sections and mounted on slides. Graded ethanol was used to rehydrate the sections after deparaffinization in xylene at room temperature. After washing with phosphate-buffered saline (PBS), the sections were placed in $3 \%$ hydrogen peroxide for $20 \mathrm{~min}$ to inhibit endogenous peroxidase. Antigen retrieval was carried out by soaking the slides in $0.01 \mathrm{M}$ citrate buffer (pH 6.0) and heating them for $30 \mathrm{~min}$ in a microwave. After incubation with a primary MMP-1 antibody (dilution 1:500; ab137332; Abcam) at $4^{\circ} \mathrm{C}$ overnight, the slides were washed with PBS and incubated with biotinylated secondary antibody for half an hour at $37^{\circ} \mathrm{C}$. 3,3-Diaminobenzidine tetrahydrochloride (DAB) was applied as a chromogen, and the sections were counterstained with hematoxylin. PBS was substituted for the primary antibody and used as a negative control.

The intensity degrees of positive signals were determined as 0 , none; 1 , weak; 2 , moderate; 3 , intense; and 4 , strongly intense; and the percentage degrees of the number of positive cells were recorded as $0,0 \% ; 1,1-25 \% ; 2,26-50 \% ; 3,51-75 \%$; and $4,76-100 \%$. The present study defined the outcome by multiplying the aforementioned two scores to achieve the final score of IHC staining. A score of $<3$ was categorized as 'negative', and a score of $\geq 3$ was categorized as 'positive' in the following statistical analysis. Two pathologists evaluated the scores separately to exclude subjectivity.

Western blot analysis. Cells were harvested, washed with PBS and lysed with radioimmunoprecipitation assay buffer (RIPA; Beyotime Institute of Biotechnology) to extract the total proteins. After determining the protein concentrations using a BCA assay kit (Thermo Fisher Scientific, Inc.), an 
equal amount of $20 \mu \mathrm{g}$ protein from each sample was resolved via $10 \%$ SDS-PAGE and then the proteins were transferred to polyvinylidene difluoride membranes (EMD Millipore). After submerging the membranes in 5\% non-fat dry milk for $2 \mathrm{~h}$ to block non-specific binding, they were incubated with a primary antibody in TBST at $4^{\circ} \mathrm{C}$ overnight. The primary antibodies used were as follows: MMP-1 (dilution 1:500; ab6721), p-Akt (dilution 1:1,000; ab64148), c-myc (dilution 1:1,000; ab32072), E-cadherin (dilution 1:1,000; ab40772), $\mathrm{N}$-cadherin (dilution 1:1,000; ab76057), vimentin (dilution 1:1,000; ab92547), Twist1 (dilution 1:1,000; ab50581), and GAPDH (dilution 1:1,000; ab9485; all from Abcam). The PVDF membranes were subsequently washed with PBS and were then incubated with a Goat Anti-Rabbit horseradish peroxidase (HRP)-conjugated secondary antibody (dilution 1:2,000; ab6721; Abcam). The proteins were developed with a chemiluminescent substrate (Thermo Fisher Scientific, Inc.), and the expression was normalized to GAPDH.

Wound healing assay. Approximately $5 \times 10^{5}$ cells were seeded into each well of 6-well plates containing complete medium and the cells were cultured overnight in an incubator set at $37^{\circ} \mathrm{C}$ and $5 \% \mathrm{CO}_{2}$. When the cell monolayers reached $100 \%$ confluence, a yellow pipette tip was used to scrape them. After washing with $1 \mathrm{X}$ PBS, the cells were placed back in the incubator. The breadth of the scratched areas was measured at 0,24, 48 and $72 \mathrm{~h}$ under a fluorescence microscope (CKX41; Olympus Corp.).

Transwell assay. To estimate the migration ability of the cells, individual groups of cells (approximately $1 \times 10^{5}$ cells/well) suspended in $200 \mu \mathrm{l}$ of serum-free medium were placed into the upper chambers of an 8- $\mu$ m-pore Transwell (Corning Inc.) coated with Matrigel matrix. All the upper chambers were placed in 24-well plates after the lower chambers were filled with $500 \mu \mathrm{l}$ of medium containing $20 \% \mathrm{FBS}$. Next, the chambers were incubated at $37^{\circ} \mathrm{C}$ in a $5 \% \mathrm{CO}_{2}$ atmosphere for $24 \mathrm{~h}$, swabbing the upper chambers with cotton swabs in succession. The cells located on the underside of the upper chambers were fixed using $4 \%$ paraformaldehyde and stained using $0.1 \%$ crystal violet at $25^{\circ} \mathrm{C}$ for $15 \mathrm{~min}$. The migrated cells were counted in five randomly selected visual fields at an x200 magnification. The same procedures were applied in the migration assay, however, the upper chambers were replaced with uncoated chambers. All assays were carried out in triplicate.

Tumorigenesis assay in vivo. A total of six female BALB/c nude mice (age 6 weeks, weight 18-22 g) were purchased from the SLAC Laboratory Animal Center and divided into two groups with three mice in each group. The use of animals was approved by the Institute Research Medical Ethics Committee of The First Affiliated Hospital of Xi'an Jiaotong University.

The SW-480 cells treated with a negative control or with an MMP-1 knockdown were harvested and inoculated into the right forelimb of nude mice $\left(1 \times 10^{6}\right.$ cells/mouse). Every week, the tumor was measured using Vernier calipers and the volume was computed by the following formula: Tumor volume $\left(\mathrm{mm}^{3}\right)=$ length $(\mathrm{mm}) \mathrm{x}$ width ${ }^{2}\left(\mathrm{~mm}^{2}\right) / 2$. All assays were carried out in triplicate. All animals were euthanized by cervical dislocation at the 3rd week. Then, the tumors were resected, weighed and fixed with paraffin for subsequent experiments.

Statistical analysis. All data were statistically analyzed using GraphPad Prism 6.0 (GraphPad Software) and SPSS 18.0 software. All in vitro assays were repeated three times, and the data are presented as the mean \pm SD. Differences between experimental groups were evaluated with Student's t-tests or one-way analysis of variance (ANOVA) followed by Fishers' least significant difference test (LSD). Statistical significance was defined as $\mathrm{P}<0.05$.

\section{Results}

MMP-1 is overexpressed in colorectal carcinomas and is related to poor prognosis in colorectal patients. Bioinformatics analyses revealed that the expression of MMP-1 was significantly increased in colorectal carcinoma samples (Fig. 1A-C). The results of immunohistochemistry revealed that the expression levels of MMP-1 protein were significantly increased in 28/49 colorectal cancer tissues compared with 11/49 adjacent non-tumor tissues (Fig. 1D and E). To further investigate the association between the expression level of MMP-1 protein and clinicopathological features, a chi-square test and a two independent samples t-test were performed to assess the relationship between MMP-1 and the clinical characteristics of colorectal cancer patients. The P-values revealed that high expression of MMP-1 was associated with the TNM stage $(\mathrm{P}<0.01)$ as well as with lymphatic metastasis $(\mathrm{P}<0.01$; Table I). These results demonstrated that increased MMP-1 expression was related to poor diagnosis in colorectal carcinoma.

In the next experiment, it was explored whether the high expression of MMP-1 influenced the prognosis of colorectal cancer patients. The outcome of Kaplan-Meier analyses revealed that a high expression level of MMP-1 was related to poor prognosis in both overall survival $(\mathrm{P}<0.01)$ and recurrence-free survival $(\mathrm{P}<0.01$; Fig. $1 \mathrm{~F}$ and $\mathrm{G})$.

Downregulation of MMP-1 inhibits cell proliferation in vitro. This study examined the expression levels of MMP-1 protein in various colorectal cancer cell lines via western blotting. Although the expression levels were variable in these cell lines, MMP-1 was expressed in most of them (Fig. 2A and B). A similar expression pattern was also revealed when measuring the mRNA of MMP-1 by means of real-time PCR. According to the aforementioned results, the HT-29 and SW-480 cell lines were selected for the next experiments.

The HT-29 and SW-480 cell lines were stably transfected with an shMMP-1 lentivirus and an empty vector as a control. To verify the efficiency of infection, real-time PCR was performed after transfection (Fig. 2C and D). To further determine the effect of transfection, the expression levels were assessed by western blotting (Fig. 2E). All of the aforementioned results revealed that the expression levels of MMP-1 protein decreased after the cells were transfected with lentivirus.

Having knocked down the expression of MMP-1 in the HT-29 and SW480 cell lines, the role of MMP-1 in the progression of colorectal carcinoma was investigated. CCK-8 assays revealed that downregulation of MMP-1 expression attenuated the 
A

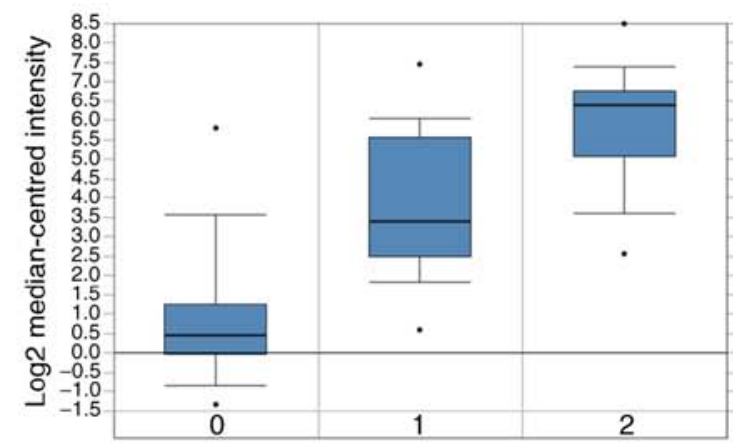

B

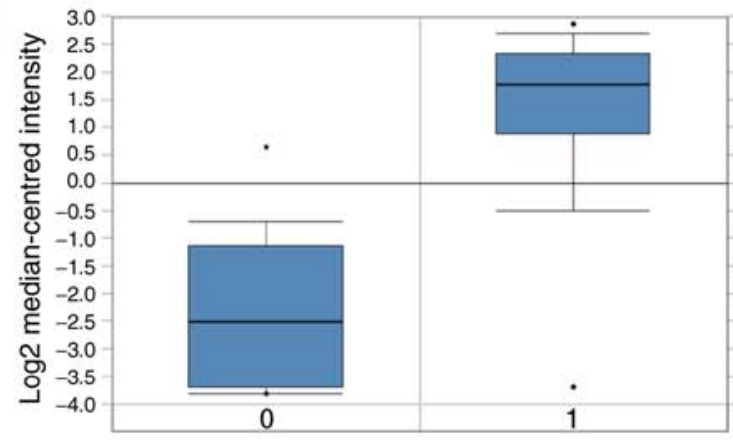

C

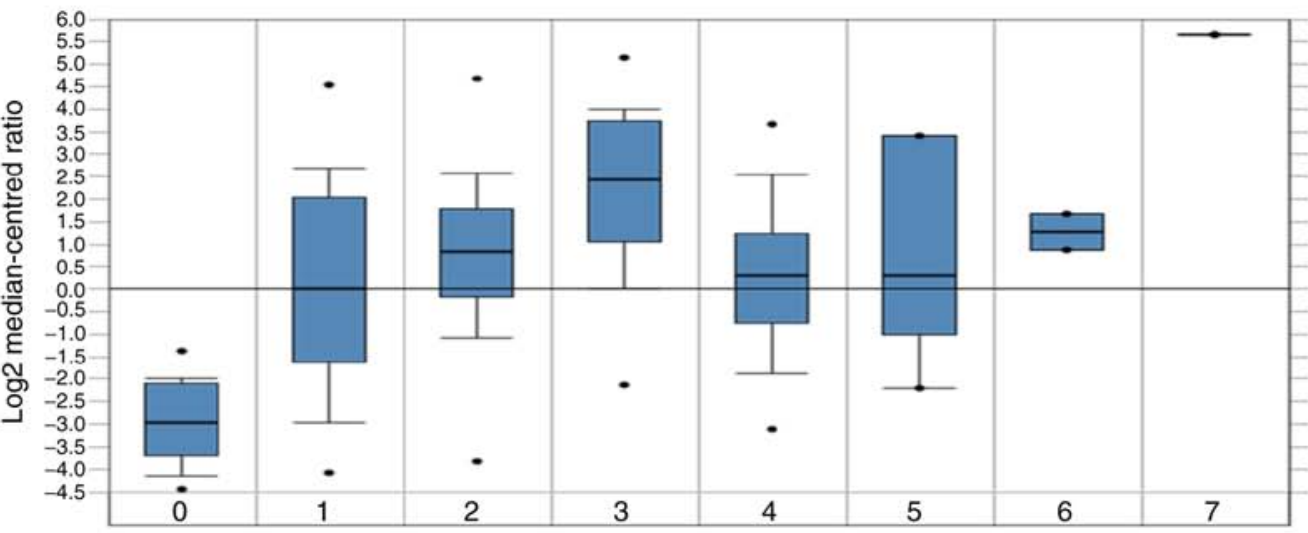

D

Normal

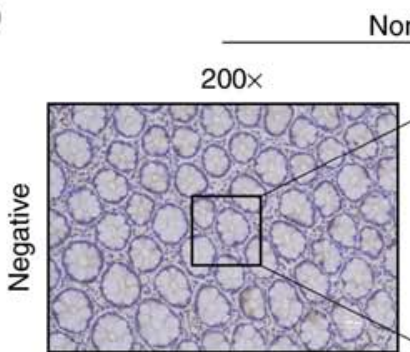

$400 \times$
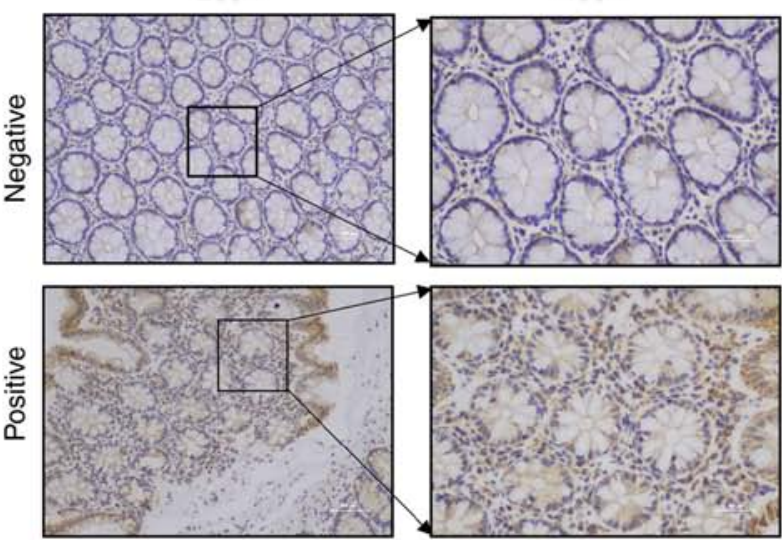

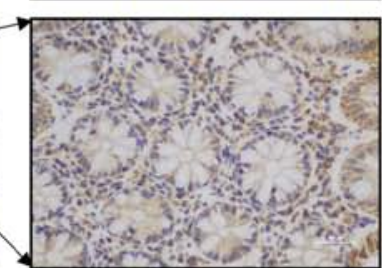

Tumour

$200 x$
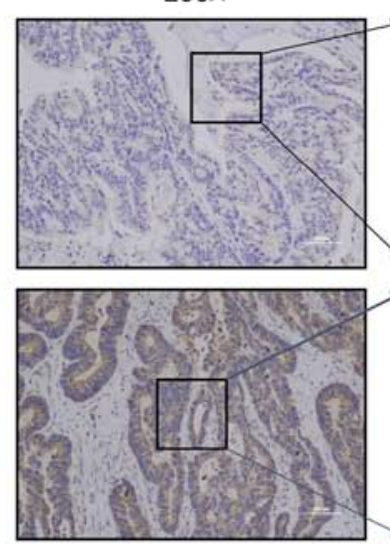

$400 \times$
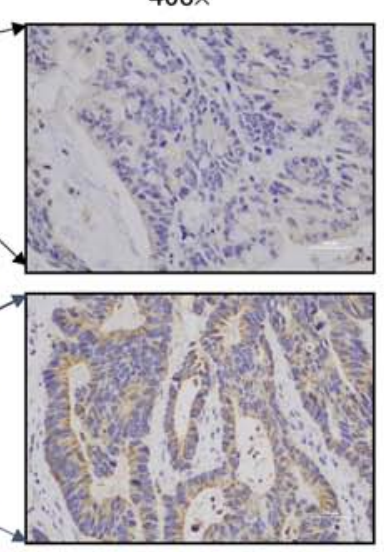

E

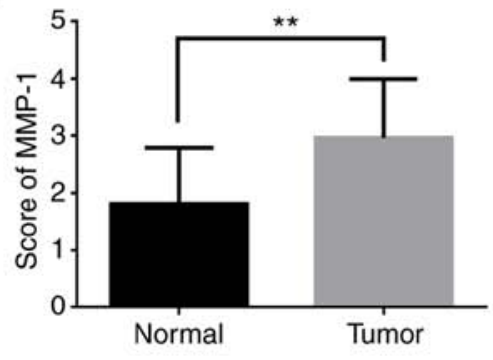

$\mathrm{F}$

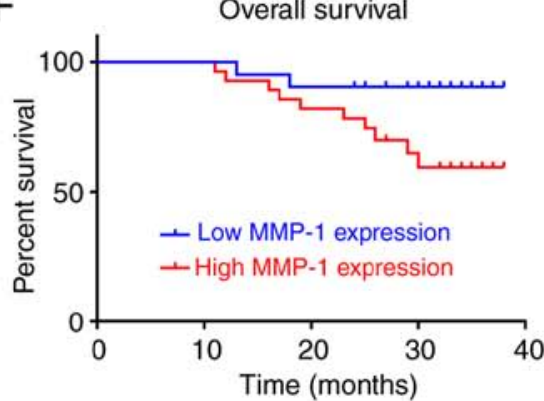

G Recurrence-free survival

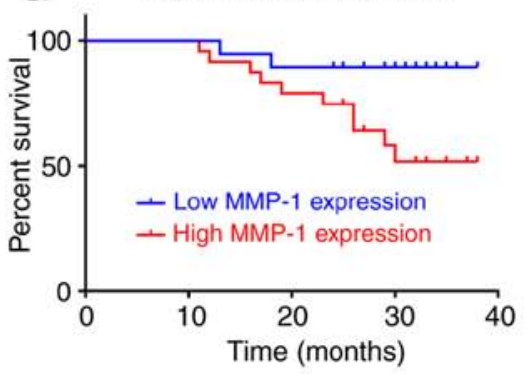

Figure 1. Expression of MMP-1 in patient samples and colorectal cancer cell lines. (A) The expression levels of MMP-1 in a first dataset, 0 represents normal tissues $(n=24), 1$ represents colorectal carcinoma $(n=36)$ and 2 represents colorectal adenocarcinoma $(n=45)$. (B) The expression levels of MMP-1 in a second dataset, 0 represents colon adenocarcinoma $(\mathrm{n}=18)$, and 1 represents the adjacent non-tumor tissues $(\mathrm{n}=18)$. (C) The expression levels of MMP-1 in a third dataset, $0-7$ represents respectively the normal tissues $(n=22)$, cecum adenocarcinoma $(n=22)$, colon adenocarcinoma $(n=101)$, colon mucinous adenocarcinoma $(n=22)$, rectal adenocarcinoma $(n=60)$, rectal mucinous adenocarcinoma $(n=6)$, rectosigmoid adenocarcinoma $(n=3)$, rectosigmoid mucinous adenocarcinoma $(\mathrm{n}=1)$. (D) Expression of MMP-1 in 49 colorectal cancer samples were assessed by IHC. Typical scans of low and high expression of MMP-1 are presented. (E) Comparison of MMP-1 expression in tumor and normal tissues by IHC score ( $\left.{ }^{* * *} \mathrm{P}<0.01\right)$. (F) Kaplan-Meier analysis of the relationship between the expression level of MMP-1 and overall survival time in colorectal cancer patients. (G) Kaplan-Meier analysis of the relationship between the expression level of MMP-1 and recurrence-free survival time in colorectal cancer patients. IHC, immunohistochemistry. MMP-1, matrix metalloproteinase-1. 
Table I. Relationship between MMP-1 and clinicopathological parameters in colorectal tumors.

\begin{tabular}{|c|c|c|c|c|}
\hline \multirow[b]{2}{*}{ Characteristics } & \multicolumn{2}{|c|}{ Low expression } & \multicolumn{2}{|c|}{ High expression } \\
\hline & $\mathrm{N}=21$ & $\mathrm{~N}=28$ & $\mathrm{t}$-test or $\chi^{2}$ & P-value \\
\hline Age $(x \pm s)$ & $56.47 \pm 14.63$ & $59.53 \pm 11.02$ & 0.835 & 0.408 \\
\hline Sex & & & 0.062 & 0.804 \\
\hline Male & 12 & 15 & & \\
\hline Female & 9 & 13 & & \\
\hline $\mathrm{BMI}\left(\mathrm{kg} / \mathrm{m}^{2}, \mathrm{x} \pm \mathrm{s}\right)$ & $22.26 \pm 2.60$ & $23.18 \pm 3.04$ & 1.113 & 0.271 \\
\hline Tumor location & & & 0.340 & 0.560 \\
\hline Colon & 11 & 17 & & \\
\hline Rectum & 10 & 11 & & \\
\hline Tumor size $(\mathrm{cm}, \mathrm{x} \pm \mathrm{s})$ & $4.21 \pm 1.53$ & $4.95 \pm 2.07$ & 1.371 & 0.177 \\
\hline Stage & & & 8.041 & 0.018 \\
\hline I & 6 & 3 & & \\
\hline II & 10 & 7 & & \\
\hline III & 5 & 18 & & \\
\hline pT status & & & 0.331 & 0.565 \\
\hline $1 / 2$ & 6 & 6 & & \\
\hline $3 / 4$ & 15 & 22 & & \\
\hline Differentiation & & & & $0.625^{\mathrm{a}}$ \\
\hline Well & 1 & 3 & & \\
\hline Moderate/Poor & 20 & 25 & & \\
\hline Vascular cancer embolus & & & 0.526 & 0.468 \\
\hline Positive & 2 & 6 & & \\
\hline Negative & 19 & 22 & & \\
\hline Lymphatic metastasis & & & 7.894 & 0.005 \\
\hline Positive & 5 & 18 & & \\
\hline Negative & 16 & 10 & & \\
\hline
\end{tabular}

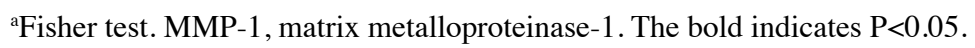

proliferative capability of colorectal cell lines (Fig. 2F and G). In addition, the number of HT-29 and SW-480 colonies was significantly decreased after the expression of MMP-1 was knocked down, indicating that MMP-1 enhances the colony formation capability of these cell lines (Fig. 2H and I).

Downregulation of MMP-1 attenuates the migration and invasion of colorectal cancer cells. Subsequently, Transwell assays were performed to evaluate the influence of MMP-1 on the invasive ability of colorectal cells. The migration and invasion experiments demonstrated that downregulated expression of MMP-1 attenuated the migratory and invasive capabilities of colorectal cell lines (Fig. 3A-C). Then, wound healing assays were carried out to measure the influence of MMP-1 on the migration capability of the cells (Fig. 3D-F). The outcomes demonstrated that silencing of MMP-1 attenuated the migration potential of colorectal cells.

Downregulation of MMP-1 inhibits cell proliferation in vivo. To further verify the tumorigenic ability of MMP-1 in vivo, mice were subcutaneously inoculated with shMMP-1-treated
HT-29 cells and control HT-29 cells. The subcutaneous tumor nodules resected from the mice with shMMP-1-treated HT-29 cells grew slower than those resected from the empty vector group (Fig. 4A-E). In addition to tumor volume and weight, the common proliferation marker, Ki-67, was assessed via western blotting. The results demonstrated the suppressed expression of $\mathrm{Ki}-67$ in tumor nodules derived from shMMP-1-treated cells compared to tumor nodules derived from control cells (Fig. 4F and G). All of the evidence revealed in Fig. 4 demonstrated that interfering with the expression of MMP-1 in colorectal cancer can inhibit the growth of tumors in vivo.

MMP-1 is involved in EMT and activation of the Akt signaling pathway in colorectal carcinoma. To reveal the latent mechanism through which MMP-1 enhances the progression of colorectal carcinoma, western blotting was used to identify the potential signaling pathway associated with the downregulation of MMP-1. This study revealed that the Akt signaling pathway was involved in both the HT-29 and SW-480 cell lines transfected with shMMP-1. The western blot results revealed that the expression of phosphorylated Akt as well as c-Myc 
A

MMP-1

GAPDH

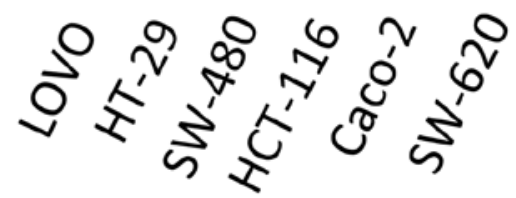

C

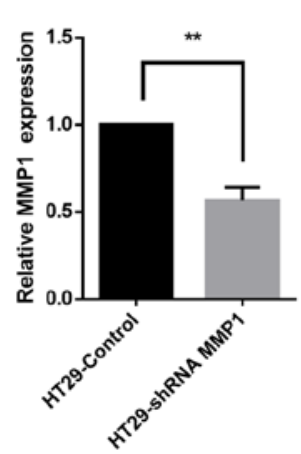

$\mathrm{F}$

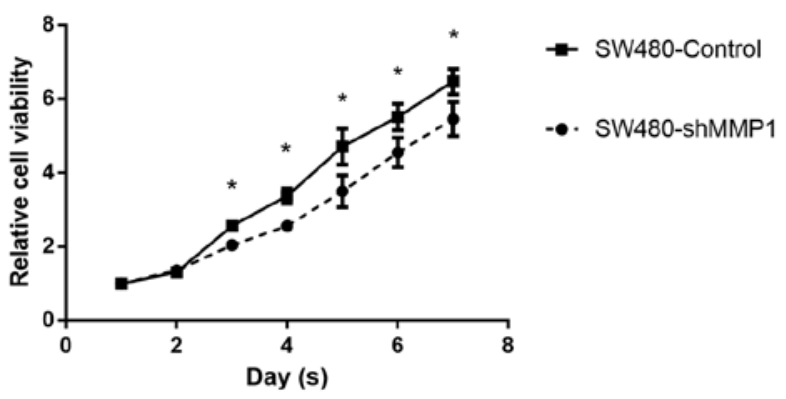

G

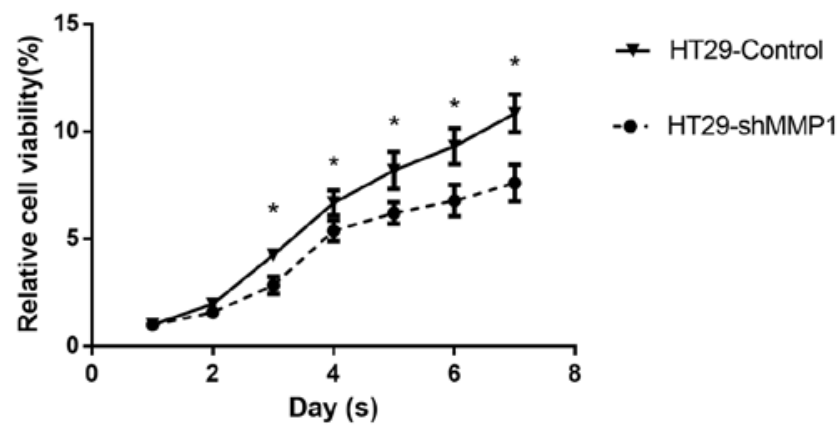

B

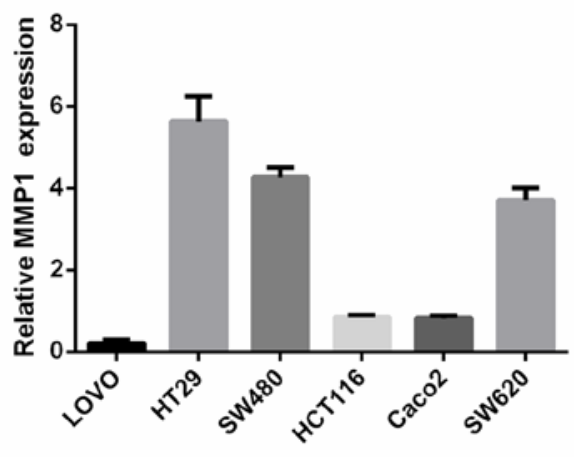

$E$ HT29 SW480

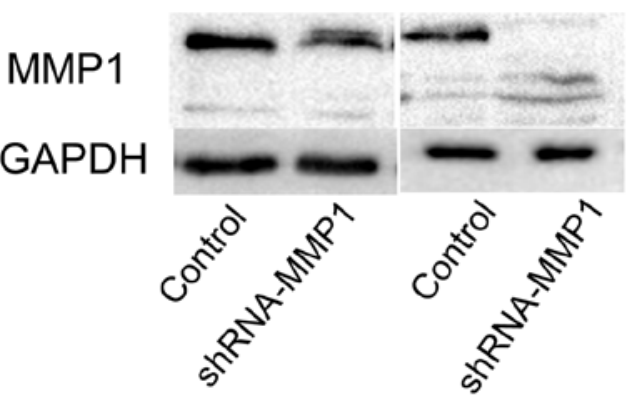

H Control shRNA-MMP1
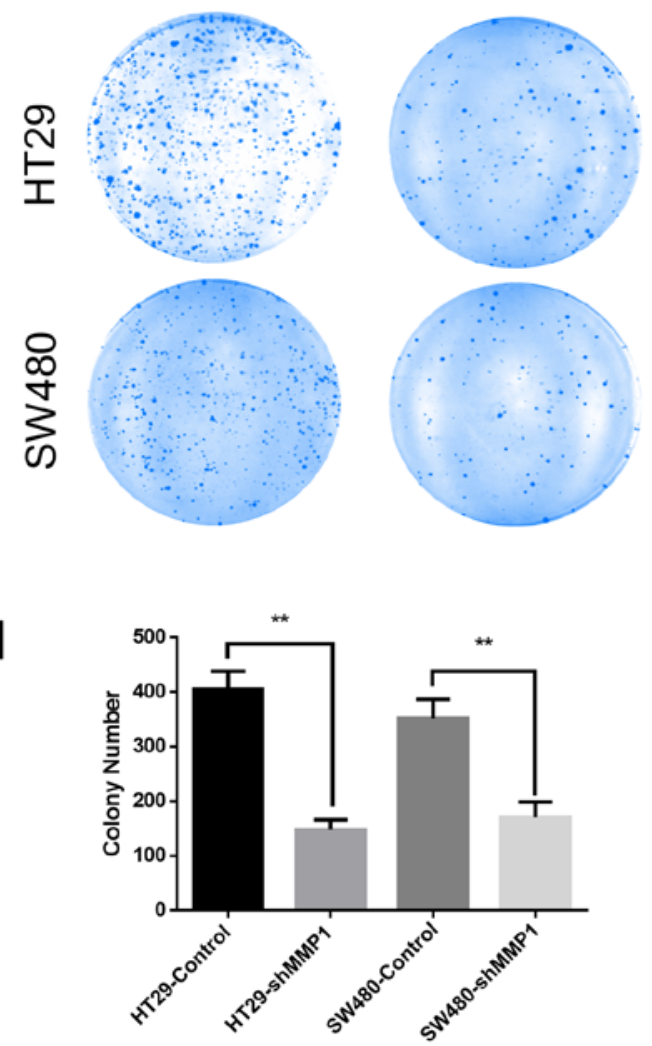

Figure 2. MMP-1 promotes proliferation of colorectal cells in vitro. (A) MMP-1 in colorectal cancer cell lines was analyzed by western blotting. (B) The expression of MMP-1 was normalized by GAPDH. (C and D) MMP-1 shRNA was stably transfected into HT-29 and SW-480 cells. The mRNA level of MMP-1 was determined by real-time PCR $\left({ }^{* *} \mathrm{P}<0.01\right)$. (E) The downregulation of MMP-1 protein expression in HT-29 and SW-480 was demonstrated by western blotting. (F and $\mathrm{G}$ ) The cell viability was assessed by CCK-8 assay after MMP-1 was consistently knocked-down ("P<0.05). (H and I) In vitro tumor growth ability of cells infected by MMP-1 shRNA and empty vector was examined by colony formation assays. The representative images and statistical data are presented. CCK-8, Cell Counting Kit-8 ( $\left.{ }^{* *} \mathrm{P}<0.01\right)$. MMP-1, matrix metalloproteinase-1. 
A

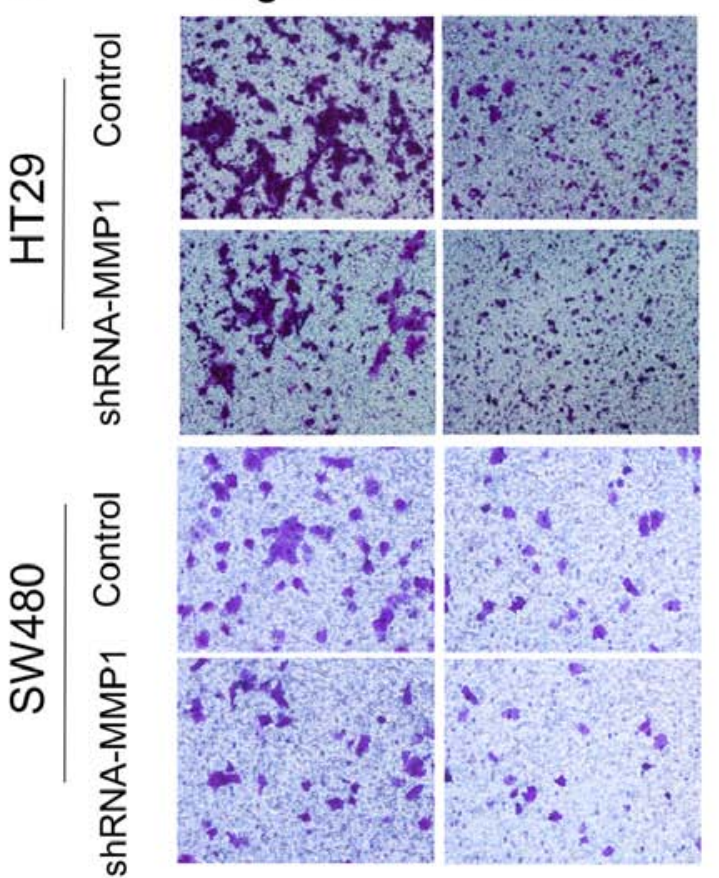

B

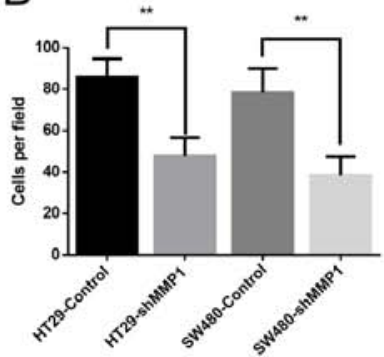

C

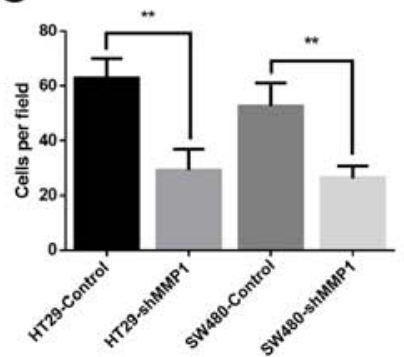

D

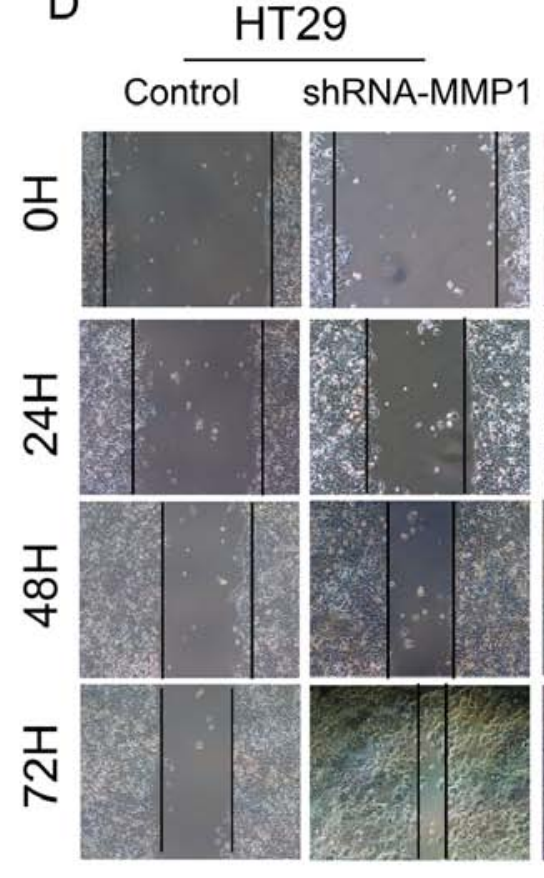

E

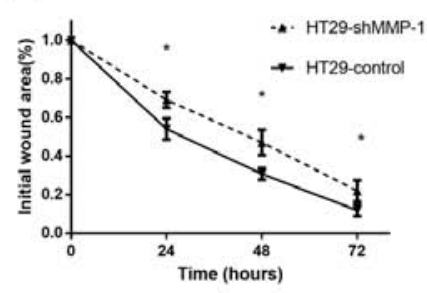

SW480

Control shRNA-MMP1
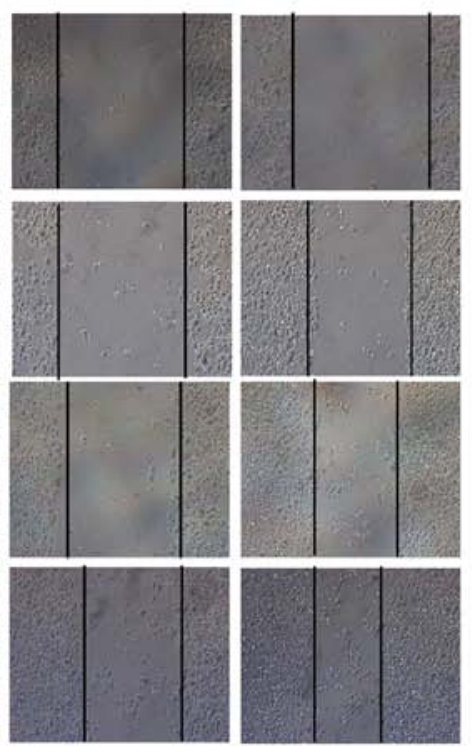

$\mathrm{F}$

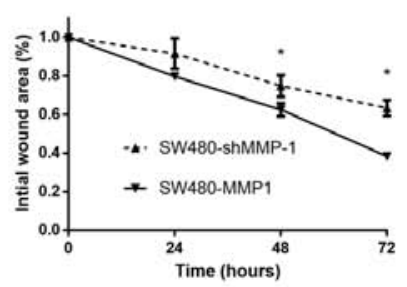

Figure 3. Decreased expression of MMP-1 attenuates the invasion and migration capability in vitro. (A) Transwell assays were used to assess the migratory and invasive abilities of cells in which the MMP-1 expression was downregulated. (B and C) Data from the Transwell migration and invasion assays are presented by bar graphs. ( $\left.{ }^{* *} \mathrm{P}<0.01\right)$. (D) The suppressed migration capability in cells stably introduced with shMMP-1 vector was demonstrated by wound healing assays ( $\mathrm{E}$ and F) Results of wound healing assays were statistically analyzed and are presented by line charts. $\left({ }^{* * *} \mathrm{P}<0.05\right)$. MMP-1, matrix metalloproteinase-1.

was suppressed after the downregulation of MMP-1 (Fig. 5), while the expression of total Akt was not significantly different (data not shown). Furthermore, the knockdown of MMP-1 suppressed EMT in colorectal carcinoma.

In HT-29 and SW-480 cells, the downregulation of MMP-1 induced a significant decrease in $\mathrm{N}$-cadherin, vimentin and Twist1 expression and a simultaneous increase in E-cadherin expression compared to cells transfected with the empty vector (Fig. 5).

Collectively, MMP-1 promoted activation of the Akt signaling pathway and EMT in colorectal carcinoma.

\section{Discussion}

At the beginning of our study, the expression of MMP-1 was analyzed in colorectal cancer patients based on datasets from the UCSC and TCGA databases, and the results revealed that MMP-1 was upregulated in colorectal cancer. Next, it was confirmed that MMP-1 was expressed in all assessed colorectal cell lines by western blotting. The immunohistochemical assessment indicated that the expression of MMP-1 had a negative relationship with prognosis in colorectal carcinoma. This result corresponded to a study by Langenskiöld et al, which demonstrated that MMP-1 predicted poor diagnosis and short-term survival in a cohort of 136 patients (27). Accumulating evidence has indicated that high expression MMP-1 predicts poor diagnosis and short-time survival not only in colorectal cancer but also in other human cancers $(26,28,29)$. Moreover, the clinicopathological parameters demonstrated that high expression of MMP-1 was related to lymphatic metastasis, which was in accordance with studies on MMP-1 in breast cancer (30), esophageal adenocarcinomas (31), oral tongue cancer (32), and oropharyngeal cancer (33).

In the present study, in vitro and in vivo experiments revealed that downregulated expression of MMP-1 attenuated the proliferation, migration and invasion capabilities of colorectal cancer cells, implicating the oncogenic significance of MMP-1 in colorectal carcinogenesis. These results indicated that MMP-1 plays a significant role in the progression of colorectal cancer. Some recent studies also indicated that MMP-1 was able to promote the proliferation, migration and invasion of cancer in vitro and in vivo. Weiss et al reported 
A
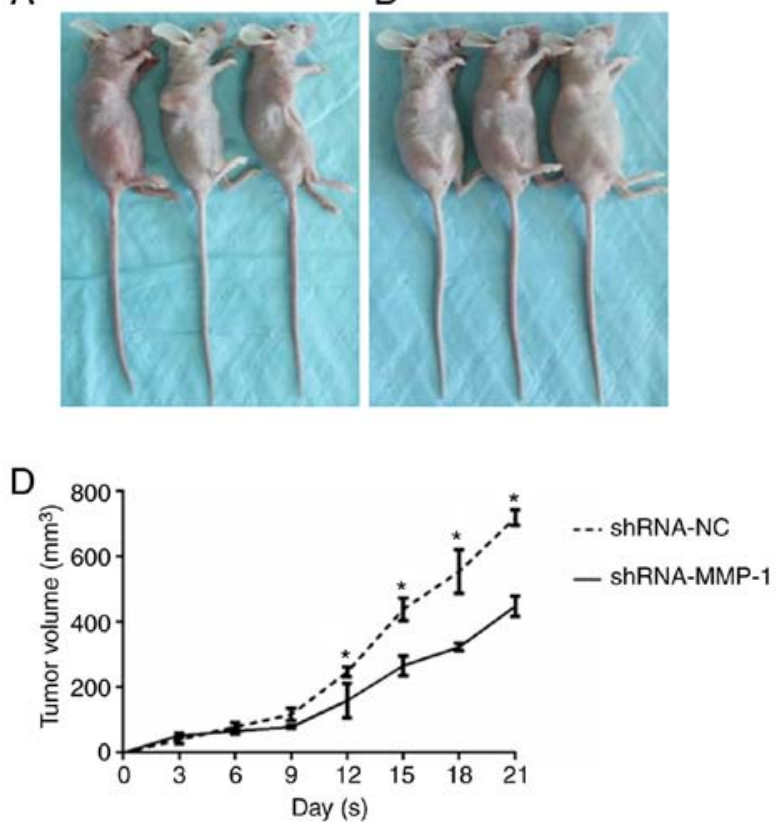

$\mathrm{F}$

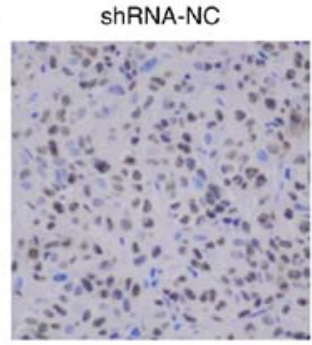

B

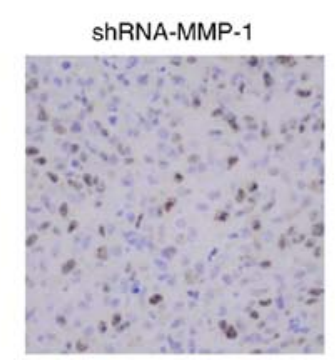

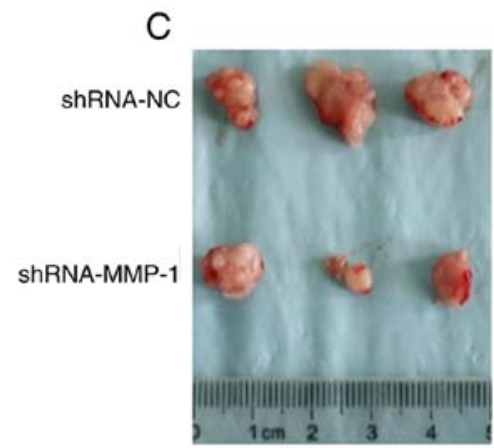

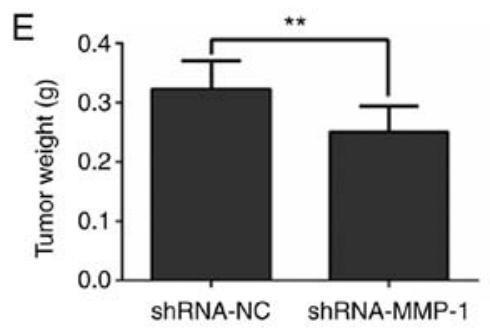

G

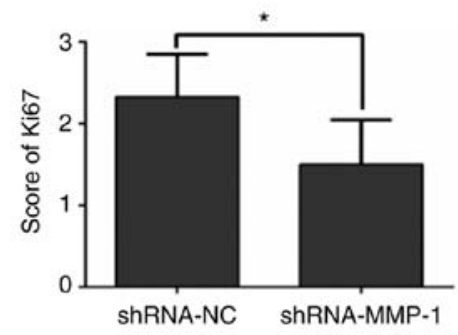

Figure 4. Xenograft models demonstrate that MMP-1 promotes colorectal tumor growth in vivo. (A-C) HT-29 cells transfected by MMP-1 shRNA or negative control were inoculated in the right flanks of nude mice. The tumor nodes were harvested at the 3rd week. (D and E) The tumor volume and weight were measured and presented by statistical graphs $\left({ }^{*} \mathrm{P}<0.05,{ }^{* *} \mathrm{P}<0.01\right)$. (F and $\left.\mathrm{G}\right)$ The expression of Ki67 in xenograft tumors was determined by IHC. The representative scan fields and statistical data are presented. IHC, immunohistochemistry ("P $<0.05)$. MMP-1, matrix metalloproteinase-1.

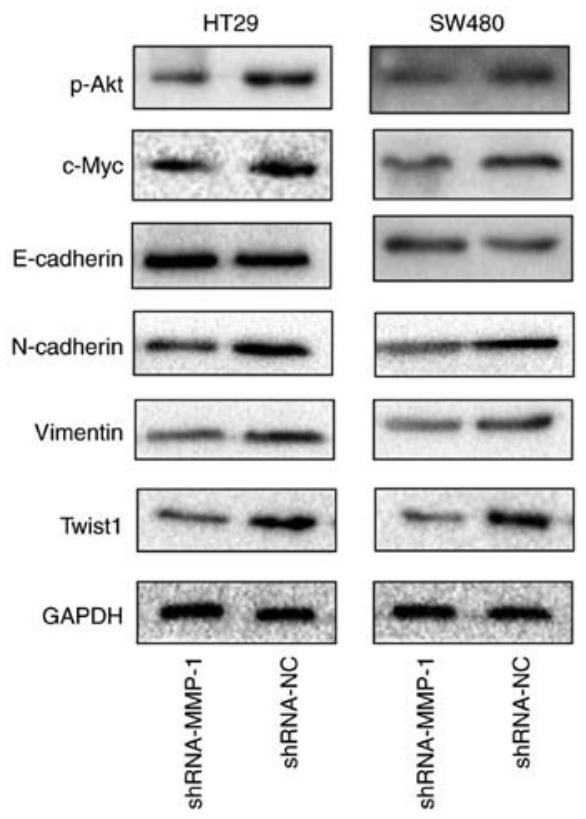

Figure 5. MMP-1 promotes the progression of colorectal cancer via EMT and the p-Akt signaling pathway. The expression of hallmarks involved in EMT and the p-Akt signaling pathway in cells transfected by MMP-1 shRNA or empty vector was detected using western blotting. EMT, epithelial-mesenchymal transition. MMP-1, matrix metalloproteinase-1. that MMP-1 participated in the proliferation of melanoma in coordination with TWIST1 in vitro (34). A study by Saito et al revealed that MMP-1 contributed to the migration of lung carcinoma via the mTOR signaling pathway in vitro (14). Furthermore, research by Fuhrman-Luck et al demonstrated that MMP-1 promoted prostate cancer bone metastases in a humanized in vivo bone metastasis model related to KLK4 (35). Evidence has revealed that increased expression of MMP-1 may serve as an oncogene in esophageal cancer, promoting the proliferation and migration of esophageal squamous cell carcinoma (26). In addition to functions in the incidence and invasion of the aforementioned cancers, MMP-1 may also regulate the early perineural invasiveness of pancreatic cancer via a PAR1/SP/NK1R paracrine loop (36). As an agonist of the protease-activated receptor-1 (PAR1), MMP-1 is propitious to the angiogenesis and permeability of blood vessels in breast cancer (37). Moreover, MMP-1 was revealed to facilitate resistance to chemotherapy as well as invasive progression of glioblastoma (38).

Akt, as one of the most famous factors in signaling pathways, has extensive involvement in the regulatory networks of cancers. Any protein targeting Akt can benefit research aiming at cancer therapy by influencing various regulatory signaling pathways. The present study revealed that the low expression 
of MMP-1 weakened the Akt signaling pathway in colorectal carcinoma, in accordance with what Liu et al reported (26). This result implicated MMP-1 as a potential therapeutic target in colorectal cancer research.

Furthermore, the present study revealed that the knockdown of MMP-1 affected the expression of E-cadherin, $\mathrm{N}$-cadherin, vimentin and Twist1, which was consistent with a previous study by Hanrahan et al on prostate cancer (39). Of these genes, E-cadherin is a well-known anti-oncogene that forms a functional complex with $\beta$-catenin (40). This complex participates in the process of intercellular adhesion in epithelial cells, securing the strength of the epithelial layer. In contrast, $\mathrm{N}$-cadherin is an invasion promoter that mediates the morphology change and renders epithelial cells more motile (41). Vimentin is an extensively expressed gene in mesenchymal cells, and it is overexpressed in a variety of cancers. A study by Satelli and Li provided evidence that vimentin promotes tumorigenesis via various signaling pathways (42). Twist is a typical gene in EMT, and its function of promoting the invasion and metastasis of colon cancer cells has been demonstrated by Wang et al (43). These results indicated that MMP-1 promoted invasion and metastasis not only by degrading the ECM but also by activating EMT.

In conclusion, the aforementioned results elucidated that MMP-1 promoted the malignant progression of colorectal cancer, which could function through EMT and the PI3K/Akt pathway.

\section{Acknowledgements}

Not applicable.

\section{Funding}

No funding was received.

\section{Availability of data and materials}

The data that support the findings of this study are available on request from the corresponding author. The data are not publicly available due to privacy or ethical restrictions.

\section{Authors' contributions}

KW performed the experiments, acquired the data and drafted the manuscript. JY and YW collected colorectal cancer samples, provided clinicopathological characteristics and assisted with the experiments. JG and ZX analyzed and interpreted the data. XS and JZ substantially contributed to the study conception and design. All authors read and approved the final manuscript and agree to be accountable for all aspects of the research, which ensures that questions about the accuracy or integrity of any part of the work are appropriately investigated and resolved.

\section{Ethics approval and consent to participate}

The study protocol was approved by the Ethics Committee of The First Affiliated Hospital of Xi'an Jiaotong University (Xi'an, China). Approval for the use of human samples was provided by the Institute Research Medical Ethics Committee of The First Affiliated Hospital of Xi'an Jiaotong University and written informed consent was obtained from all patients. The use of animals was approved by the Institute Research Medical Ethics Committee of The First Affiliated Hospital of Xi'an Jiaotong University.

\section{Patient consent for publication}

Not applicable.

\section{Competing interests}

The authors declare that they have no competing interests.

\section{References}

1. Bray F, Ferlay J, Soerjomataram I, Siegel RL, Torre LA and Jemal A: Global cancer statistics 2018: GLOBOCAN estimates of incidence and mortality worldwide for 36 cancers in 185 countries. CA Cancer J Clin 68: 394-424, 2018.

2. Siegel RL, Miller KD and Jemal A: Cancer statistics, 2019. CA Cancer J Clin 69: 7-34, 2019.

3. Chen W, Zheng R, Baade PD, Zhang S, Zeng H, Bray F, Jemal A, Yu XQ and He J: Cancer statistics in China, 2015. CA Cancer J Clin 66: 115-132, 2016.

4. Favoriti P, Carbone G, Greco M, Pirozzi F, Pirozzi RE and Corcione F: Worldwide burden of colorectal cancer: A review. Updates Surg 68: 7-11, 2016.

5. Singh D, Srivastava SK, Chaudhuri TK and Upadhyay G: Multifaceted role of matrix metalloproteinases (MMPs). Front Mol Biosci 2: 19, 2015.

6. Gialeli C, Theocharis AD and Karamanos NK: Roles of matrix metalloproteinases in cancer progression and their pharmacological targeting. FEBS J 278: 16-27, 2011.

7. Page-McCaw A, Ewald AJ and Werb Z: Matrix metalloproteinases and the regulation of tissue remodelling. Nat Rev Mol Cell Biol 8: 221-233, 2007.

8. McCawley LJ and Matrisian LM: Matrix metalloproteinases: They're not just for matrix anymore. Curr Opin Cell Biol 13: 534-540, 2001.

9. Shintani T, Kusuhara Y, Daizumoto K, Dondoo TO, Yamamoto H, Mori H, Fukawa T, Nakatsuji H, Fukumori T, Takahashi $\mathrm{M}$ and Kanayama $\mathrm{H}$ : The involvement of hepatocyte growth Factor-MET-Matrix metalloproteinase 1 signaling in bladder cancer invasiveness and proliferation. Effect of the MET inhibitor, Cabozantinib (XL184), on bladder cancer cells. Urology 101: 169.e7-169.e13, 2017.

10. Wang J, Liu D, Zhou W, Wang M, Xia W and Tang Q: Prognostic value of matrix metalloprotease-1/protease-activated receptor-1 axis in patients with prostate cancer. Med Oncol 31: 968, 2014.

11. Kim M, Kim HJ, Choi BY, Kim JH, Song KS, Noh SM, Kim JC, Han DS, Kim SY and Kim YS: Identification of potential serum biomarkers for gastric cancer by a novel computational method, multiple normal tissues corrected differential analysis. Clin Chim Acta 413: 428-433, 2012.

12. Yamamoto H, Itoh F, Iku S, Adachi Y, Fukushima H, Sasaki S, Mukaiya M, Hirata K and Imai K: Expression of matrix metalloproteinases and tissue inhibitors of metalloproteinases in human pancreatic adenocarcinomas: Clinicopathologic and prognostic significance of matrilysin expression. J Clin Oncol 19: 1118-1127, 2001.

13. Pittayapruek P, Meephansan J, Prapapan O, Komine M and Ohtsuki M: Role of matrix metalloproteinases in photoaging and photocarcinogenesis. Int J Mol Sci 17: pii: E868, 2016.

14. Saito R, Miki Y, Ishida N, Inoue C, Kobayashi M, Hata S, Yamada-Okabe H, Okada Y and Sasano H: The significance of MMP-1 in EGFR-TKI-resistant lung adenocarcinoma: Potential for therapeutic targeting. Int J Mol Sci 19: pii: E609, 2018.

15. Bendardaf R, Buhmeida A, Ristamäki R, Syrjänen K and Pyrhönen S: MMP-1 (collagenase-1) expression in primary colorectal cancer and its metastases. Scand J Gastroenterol 42: 1473-1478, 2007. 
16. Shiozawa J, Ito M, Nakayama T, Nakashima M, Kohno S and Sekine I: Expression of matrix metalloproteinase-1 in human colorectal carcinoma. Mod Pathol 13: 925-933, 2000.

17. Gurzu S, Silveanu C, Fetyko A, Butiurca V, Kovacs Z and Jung I: Systematic review of the old and new concepts in the epithelial-mesenchymal transition of colorectal cancer. World J Gastroenterol 22: 6764-675, 2016.

18. Thiery JP, Acloque H, Huang RY and Nieto MA: Epithelial-mesenchymal transitions in development and disease. Cell 139: 871-890, 2009.

19. Prieto-García E, Díaz-García CV, García-Ruiz I and Agulló-Ortuño MT: Epithelial-to-mesenchymal transition in tumor progression. Med Oncol 34: 122, 2017.

20. Sakamoto T, Kawano S, Matsubara R, Goto Y, Jinno T, Maruse Y, Kaneko N, Hashiguchi Y, Hattori T, Tanaka S, et al: Critical roles of Wnt5a-Ror2 signaling in aggressiveness of tongue squamous cell carcinoma and production of matrix metalloproteinase-2 via $\Delta \mathrm{Np} 63 \beta$-mediated epithelial-mesenchymal transition. Oral Oncol 69: 15-25, 2017.

21. Jiang Y, Jiao Y, Liu Y, Zhang M, Wang Z, Li Y, Li T, Zhao X and Wang D: Sinomenine hydrochloride inhibits the metastasis of human glioblastoma cells by suppressing the expression of matrix Metalloproteinase-2/-9 and reversing the endogenous and exogenous Epithelial-mesenchymal transition. Int J Mol Sci 9: pii: E844, 2018.

22. Lee H, Ko JH, Baek SH, Nam D, Lee SG, Lee J, Yang WM, Um JY, Kim SH, Shim BS and Ahn KS: Embelin inhibits invasion and migration of MDA-MB-231 breast cancer cells by suppression of CXC chemokine receptor 4, matrix Metalloproteinases-9/2, and Epithelial-mesenchymal transition. Phytother Res 30: 1021-1032, 2016.

23. Chatterjee K, Jana S, DasMahapatra P and Swarnakar S: EGFR-mediated matrix metalloproteinase-7 up-regulation promotes epithelial-mesenchymal transition via ERK1-AP1 axis during ovarian endometriosis progression. FASEB J 32: 4560-4572, 2018.

24. Zhao XL, Sun T, Che N, Sun D, Zhao N, Dong XY, Gu Q, Yao Z and Sun BC: Promotion of hepatocellular carcinoma metastasis through matrix metalloproteinase activation by epithelial-mesenchymal transition regulator Twist1. J Cell Mol Med 15: 691-700, 2011.

25. Chen GH, Huang CH, Luo HY and Jiang ST: Effect of small interfering RNAs on matrix metalloproteinase 1 expression. Biotechnol Rep (Amst) 4: 5-13, 2014.

26. Liu M, Hu Y, Zhang MF, Luo KJ, Xie XY, Wen J, Fu JH and Yang H: MMP1 promotes tumor growth and metastasis in esophageal squamous cell carcinoma. Cancer Lett 377: 97-104, 2016.

27. Langenskiöld M, Ivarsson ML, Holmdahl L, Falk P, Kåbjörn-Gustafsson C and Angenete E: Intestinal mucosal MMP-1-a prognostic factor in colon cancer. Scand J Gastroenterol 48: 563-569, 2013

28. Bianco BC, Scotti FM, Vieira DS, Biz MT, Castro RG and ModoloF: Immunohistochemical expression of matrix metalloproteinase-1, matrix metalloproteinase-2 and matrix metalloproteinase-9, myofibroblasts and Ki-67 in actinic cheilitis and lip squamous cell carcinoma. Int J Exp Pathol 96: 311-318, 2015.

29. Wang QM, Lv L, Tang Y, Zhang L and Wang LF: MMP-1 is overexpressed in triple-negative breast cancer tissues and the knockdown of MMP-1 expression inhibits tumor cell malignant behaviors in vitro. Oncol Lett 17: 1732-1740, 2019.
30. Eiró N, González LO, Atienza S, González-Quintana JM, Beridze N, Fernandez-Garcia B, Pérez-Fernández R, García-Caballero T, Schneider J and Vizoso FJ: Prediction of metastatic breast cancer in non-sentinel lymph nodes based on metalloprotease-1 expression by the sentinel lymph node. Eur J Cancer 49: 1009-1017, 2013.

31. Grimm M, Lazariotou M, Kircher S, Stuermer L, Reiber C, Höfelmayr A, Gattenlöhner S, Otto C, Germer CT and von Rahden BH: MMP-1 is a (pre-)invasive factor in Barrett-associated esophageal adenocarcinomas and is associated with positive lymph node status. J Transl Med 8: 99, 2010.

32. Zhang Z, Pan J, Li L, Wang Z, Xiao W and Li N: Survey of risk factors contributed to lymphatic metastasis in patients with oral tongue cancer by immunohistochemistry. J Oral Pathol Med 40: 127-134, 2011.

33. Lassig AAD, Joseph AM, Lindgren BR and Yueh B: Association of oral cavity and oropharyngeal cancer biomarkers in surgical drain fluid with patient outcomes. JAMA Otolaryngol Head Neck Surg 143: 670-678, 2017.

34. Weiss MB, Abel EV, Mayberry MM, Basile KJ, Berger AC and Aplin AE: TWIST1 is an ERK1/2 effector that promotes invasion and regulates MMP-1 expression in human melanoma cells. Cancer Res 72: 6382-6392, 2012.

35. Fuhrman-Luck RA, Stansfield SH, Stephens CR, Loessner D and Clements JA: Prostate Cancer-associated Kallikrein-related peptidase 4 activates matrix Metalloproteinase-1 and Thrombospondin-1. J Proteome Res 15: 2466-2478, 2016.

36. Huang C, Li Y, Guo Y, Zhang Z, Lian G, Chen Y, Li J, Su Y, Li J, Yang K, et al: MMP1/PAR1/SP/NK1R paracrine loop modulates early perineural invasion of pancreatic cancer cells. Theranostics 8: 3074-3086, 2018.

37. Juncker-Jensen A, Deryugina EI, Rimann I, Zajac E, Kupriyanova TA, Engelholm LH and Quigley JP: Tumor MMP-1 activates endothelial PAR1 to facilitate vascular intravasation and metastatic dissemination. Cancer Res 73: 4196-4211, 2013.

38. Dong F,Eibach M,Bartsch JW, Dolga AM,Schlomann U,Conrad C, Schieber S, Schilling O, Biniossek ML, Culmsee C, et al: The metalloprotease-disintegrin ADAM8 contributes to temozolomide chemoresistance and enhanced invasiveness of human glioblastoma cells. Neuro Oncol 17: 1474-1485, 2015.

39. Hanrahan K, O'Neill A, Prencipe M, Bugler J, Murphy L, Fabre A, Puhr M, Culig Z, Murphy K and Watson RW: The role of epithelial-mesenchymal transition drivers ZEB1 and ZEB2 in mediating Docetaxel-resistant prostate cancer. Mol Oncol 11: 251-265, 2017.

40. Pal I, Rajesh Y, Banik P, Dey G, Dey KK, Bharti R, Naskar D, Chakraborty S, Ghosh SK, Das SK, et al: Prevention of epithelial to mesenchymal transition in colorectal carcinoma by regulation of the E-cadherin- $\beta$-catenin-vinculin axis. Cancer Lett 452 : 254-263, 2019.

41. Derycke LD and Bracke ME: N-cadherin in the spotlight of cell-cell adhesion, differentiation, embryogenesis, invasion and signalling. Int J Dev Biol 48: 463-476, 2004.

42. Satelli A and Li S: Vimentin in cancer and its potential as a molecular target for cancer therapy. Cell Mol Life Sci 68: 3033-3046, 2011.

43. Wang D, Rai B, Qi F, Liu T, Wang J, Wang X and Ma B: Influence of the Twist gene on the invasion and metastasis of colon cancer. Oncol Rep 39: 31-44, 2018.

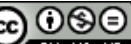

This work is licensed under a Creative Commons Attribution-NonCommercial-NoDerivatives 4.0 International (CC BY-NC-ND 4.0) License. 